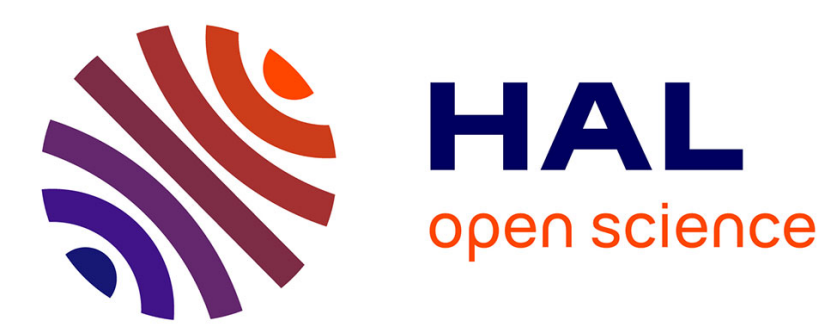

\title{
Wirtinger-based integral inequality: Application to time-delay systems
}

Alexandre Seuret, Frédéric Gouaisbaut

\section{To cite this version:}

Alexandre Seuret, Frédéric Gouaisbaut. Wirtinger-based integral inequality: Application to timedelay systems. Automatica, 2013, 49 (9), pp.2860-2866. hal-00855159

\section{HAL Id: hal-00855159 \\ https://hal.science/hal-00855159}

Submitted on 29 Aug 2013

HAL is a multi-disciplinary open access archive for the deposit and dissemination of scientific research documents, whether they are published or not. The documents may come from teaching and research institutions in France or abroad, or from public or private research centers.
L'archive ouverte pluridisciplinaire HAL, est destinée au dépôt et à la diffusion de documents scientifiques de niveau recherche, publiés ou non, émanant des établissements d'enseignement et de recherche français ou étrangers, des laboratoires publics ou privés. 


\title{
Wirtinger-Based Integral Inequality: Application to Time-Delay Systems
}

\author{
A. Seuret ${ }^{a, b}$ F. Gouaisbaut ${ }^{a, c}$ \\ ${ }^{\text {a } C N R S, ~ L A A S, ~} 7$ avenue du Colonel Roche, 31077 Toulouse, France. \\ (e-mail: aseuret,fgouaisb@laas.fr). \\ ${ }^{\mathrm{b}}$ Univ de Toulouse, LAAS, F-31400 Toulouse, France \\ ${ }^{\mathrm{c}}$ Univ de Toulouse, UPS, LAAS, F-31400, Toulouse, France.
}

\begin{abstract}
In the last decade, the Jensen inequality has been intensively used in the context of time-delay or sampled-data systems since it is an appropriate tool to derive tractable stability conditions expressed in terms linear matrix inequalities (LMI). However, it is also well-known that this inequality introduces an undesirable conservatism in the stability conditions and looking at the literature, reducing this gap is a relevant issue and always an open problem. In this paper, we propose an alternative inequality based on the Fourier Theory, more precisely on the Wirtinger inequalities. It is shown that this resulting inequality encompasses the Jensen one and also leads to tractable LMI conditions. In order to illustrate the potential gain of employing this new inequality with respect to the Jensen one, two applications on time-delay and sampled-data stability analysis are provided.
\end{abstract}

Key words: Jensen Inequality, stability analysis, time-delay systems, sampled-data systems

\section{Introduction}

The last decade has shown an increasing research activity on time-delay and/or sampled-data systems analysis and control due to both emerging adapted theoretical tools and also practical issues in the engineering field and information technology (see [? ? ]) and references therein). In the case of linear systems, many techniques allow to derive efficient criteria proving the stability of such systems. Among them, two frameworks, different in their spirits have been recognized as a powerful methodologies. The first one relies on Robust Analysis. In this framework, the time delay/sampled data system is transformed into a closed loop between a stable nominal system and a perturbation element depending either on the delay or the sampling process (which is also modeled by a time varying delay). The perturbation element is then embedded into some norm-bounded uncertainties and the use of scaled small gain theorem [? ?], Integral Quadratic Constraints (IQC) [? ], or quadratic separation approach [?] allows then to derive efficient stability criteria. The challenge is then to reduce the conservatism either by constructing elaborated interconnections which generally include state augmentation [? ] or by using finer $L_{2}$ induced norm upperbound evaluation [?], often based on Cauchy-Schwartz inequality [? ]. An- other popular approach is the use of a Lyapunov function to prove stability. For sampled-data systems, two approaches have been successfully proposed lately. The first one relies on impulsive systems and some piecewise linear Lyapunov functions [? ?]. This approach has been then extended by considering discontinuous Lyapunov functions which allow to consider aperiodic sampling [? ? ]. In the second approach, the sampled state is modeled by a time varying delayed state. In that case, the original system is recasted into a time varying delay system where Lyapunov-Krasovskii functionals [? ] can be used directly.

Hence, for sampled-data and time delay systems, the last decade has seen a tremendous emergence of research devoted to the construction of Lyapunov-Krasovskii functionals which aims at reducing the inherent conservatism of this approach. Several attempts have been done concerning the structure of the functional by extending state based Lyapunov-Krasovskii functionals ([? ? ] ), discretized Lyapunov functionals ([?]) or discontinuous Lyapunov functions [?]. Apart the choice of the functional, an important source of conservatism relies also on the way to bound some cross terms arisen when manipulating the derivative of the Lyapunov-Krasovskii functional. According to the literature on this subject (see [? ? ? ] for some recent papers), a common feature of all 
these techniques is the use of slack variables and more or less refined Jensen inequality [? ? ?]. At this point, it is clear that for both frameworks - Robust Analysis and Lyapunov functionals-, a part of the conservatism comes from the use of Jensen inequality [?], usually used to get tractable criteria. Based on this observation, the objective of the present paper is then to show how to use another class of inequalities called Wirtinger inequalities, which are well known in Fourier Analysis. Notice that this class of inequalities has been recently used to exhibit new Lyapunov functionals to prove stability of sampleddata systems [?]. In the present paper, contrary to the work of [?], we do not construct some new Lyapunov functionals. We aim rather at developing a new inequality used to reduce the conservatism when computing the derivative of Lyapunov-Krasovskii functionals. The Wirtinger inequality allows to consider a more accurate integral inequality which encompasses the Jensen one. The resulting inequality depends not only on the state $x(t)$ and the delayed or sampled state but also on the integral of the state over the delay or sampling interval. This new signal is then directly integrated into a suitable Lyapunov function, highlighting so the features of Wirtinger inequality. Hence, it results new stability criteria for time-delay systems and sampled-data systems directly expressed in terms of LMIs.

Notations: Throughout the paper $\mathbb{R}^{n}$ denotes the $n$ dimensional Euclidean space, $\mathbb{R}^{n \times m}$ is the set of all $n \times m$ real matrices. The notation $P \succ 0$, for $P \in \mathbb{R}^{n \times n}$, means that $P$ is symmetric and positive definite. The notation $\left[\begin{array}{ll}A & B \\ * & C\end{array}\right]$ stands for $\left[\begin{array}{cc}A & B \\ B^{T} & C\end{array}\right]$. For any square matrices $A$ and $B$, define $\operatorname{diag}(A, B)=\left[\begin{array}{cc}A & 0 \\ * & B\end{array}\right]$. Moreover, for any square matrix $A \in \mathbb{R}^{n \times n}$, we define $\operatorname{He}(A)=A+A^{T}$. The notation $I$ stands for the identity matrix.

\section{Preliminaries}

\subsection{Necessity of integral inequalities}

Diverse methodss are provided in the literature to assess stability of time-delay systems using LyapunovKrasovskii functionals. Among them, one of the most relevant term introduced for the first time in [?] is

$$
V\left(x_{t}\right)=\int_{t-h}^{t} \int_{s}^{t} \dot{x}^{T}(\theta) R \dot{x}(\theta) \mathrm{d} \theta \mathrm{d} s
$$

where $x$ represents the state of a time-delay system, $R \succ$ 0 and $h>0$. Differentiating this term with respect to the time variable $t$, we get

$$
\dot{V}\left(x_{t}\right)=h \dot{x}^{T}(t) R \dot{x}(t)-\int_{t-h}^{t} \dot{x}^{T}(s) R \dot{x}(s) \mathrm{d} s .
$$

The main issue related to (2) is that the integral is not appropriate to the "LMIzation" process. It consists in transforming the previous expression into an appropriate form to derive an LMI formulation of the stability conditions. In the following, the problem under consideration is to providing a new lower bound of integral quadratic terms of the form

$$
\mathcal{I}_{R}(\omega)=\int_{a}^{b} \omega^{T}(u) R \omega(u) \mathrm{d} u
$$

where $-\infty<a<b<+\infty$ are scalars, $\omega$ is a continuous function from $[a, b] \rightarrow \mathbb{R}^{n}$ and, consequently integrable.

The first method to treat this problem is based on the Jensen inequality formulated in the next lemma

Lemma 1 For a given $n \times n$-matrix $R \succ 0$ and for all continuous functions $\omega$ in $[a, b] \rightarrow \mathbb{R}^{n}$, the following inequality holds:

$$
\mathcal{I}_{R}(\omega) \geq \frac{1}{b-a}\left(\int_{a}^{b} \omega^{T}(u) \mathrm{d} u\right) R\left(\int_{a}^{b} \omega(u) \mathrm{d} u\right)
$$

The proof is omitted and can be found in [?]. Naturally, the Jensen inequality is likely to entail some inherent conservatism and several works have been devoted to the reduction of such a gap [? ? ]. In the present paper, we propose to use a different class of inequalities called Wirtinger inequalities to obtain a more accurate lower bound of this integral.

\subsection{Wirtinger inequality}

In the literature [?], Wirtinger inequalities refer to inequalities which estimate the integral of the derivative function with the help of the integral of the function. Often proved using Fourier theory, it exists several versions which depend on the characteristics or constraints we impose on the function. Let us focus on the following inequality adapted to our purpose.

Lemma 2 Consider a given $n \times n$-matrix $R \succ 0$. Then, for all function $z$ in $\mathcal{C}^{1}\left([a, b] \rightarrow \mathbb{R}^{n}\right)$ which satisfies $z(a)=z(b)=0$, the following inequality holds

$$
\int_{a}^{b} \dot{z}^{T}(u) R \dot{z}(u) \mathrm{d} u \geq \frac{\pi^{2}}{(b-a)^{2}} \int_{a}^{b} z^{T}(u) R z(u) \mathrm{d} u,
$$

Proof: The proof is omitted but can be found in [?]. $\diamond$ It is worth noting that (4) is not related to the Jensen inequality in its essence. Indeed, the function $z$ has to meet several constraints whereas the function $\omega$ is assumed to be a continuous function in the Jensen inequality. 


\subsection{Reciprocally convex combination inequality}

Recall a useful lemma inspired from the reciprocally convex combination lemma provided in [?].

Lemma $3[?]$ For given positive integers $n, m$, a scalar $\alpha$ in the interval $(0,1)$, a given $n \times n$-matrix $R \succ 0$, two matrices $W_{1}$ and $W_{2}$ in $\mathbb{R}^{n \times m}$. Define, for all vector $\xi$ in $\mathbb{R}^{m}$, the function $\Theta(\alpha, R)$ given by:

$$
\Theta(\alpha, R)=\frac{1}{\alpha} \xi^{T} W_{1}^{T} R W_{1} \xi+\frac{1}{1-\alpha} \xi^{T} W_{2}^{T} R W_{2} \xi
$$

Then, if there exists a matrix $X$ in $\mathbb{R}^{n \times n}$ such that $\left[\begin{array}{cc}R & X \\ * & R\end{array}\right] \succ 0$, then the following inequality holds

$$
\min _{\alpha \in(0,1)} \Theta(\alpha, R) \geq\left[\begin{array}{l}
W_{1} \xi \\
W_{2} \xi
\end{array}\right]^{T}\left[\begin{array}{cc}
R & X \\
* & R
\end{array}\right]\left[\begin{array}{l}
W_{1} \xi \\
W_{2} \xi
\end{array}\right]
$$

Proof: The proof is omitted but can be found in [?]. $\diamond$ This lemma will be useful to derive stability conditions for linear systems with time-varying delays.

\section{Application of the Wirtinger inequality}

The objective of this section is to provide an inequality based on Lemma 2, which, on the first hand, is implementable into a convex optimization scheme and, on the other hand, which reduces the conservatism of the Jensen inequality. To do so, the function $z$ has to be constructed such that $\mathcal{I}_{R}(\omega)$ appears naturally in the future developments. Thus a necessary condition is that $z(u)=\int_{a}^{u} \omega(s) \mathrm{d} s-y(u)$, where $y$ is a continuous function in $[a, b] \rightarrow \mathbb{R}^{n}$ to be defined.

Corollary 4 Consider a given matrix $R \succ 0$. Then, for all continuous function $\omega$ in $[a, b] \rightarrow \mathbb{R}^{n}$ the following inequality holds:

$$
\begin{aligned}
\mathcal{I}_{R}(\omega) \geq & \frac{1}{b-a}\left(\int_{a}^{b} \omega(u) \mathrm{d} u\right)^{T} R\left(\int_{a}^{b} \omega(u) \mathrm{d} u\right) \\
& +\frac{3}{b-a} \Omega^{T} R \Omega,
\end{aligned}
$$

where $\Omega=\int_{a}^{b} \omega(s) \mathrm{d} s-\frac{2}{b-a} \int_{a}^{b} \int_{a}^{s} \omega(r) \mathrm{d} r \mathrm{~d} s$.

Proof : For any continuous function $\omega$ and which admits a continuous derivative, define the function $z$ given, for all $u \in[a, b]$, by

$z(u)=\int_{a}^{u} \omega(s) \mathrm{d} s-\frac{u-a}{b-a} \int_{a}^{b} \omega(s) \mathrm{d} s-\frac{(b-u)(u-a)}{(b-a)^{2}} \Theta$, where $\Theta$ is a constant vector of $\mathbb{R}^{n}$ to be defined. The difference between this function $z$ and the one proposed in [? ] remains in the addition of the third term. By construction, the function $z$ satisfies the conditions of the Wirtinger inequality given in Lemma 2, that is $z(a)=$ $z(b)=0$. The computation of the left-hand-side of the inequality stated in Lemma 2 leads to:

$$
\begin{aligned}
\int_{a}^{b} \dot{z}^{T}(u) & R \dot{z}(u) \mathrm{d} u=\int_{a}^{b} \omega^{T}(u) R \omega(u) \mathrm{d} u \\
& -\frac{1}{b-a}\left(\int_{a}^{b} \omega(u) \mathrm{d} u\right)^{T} R\left(\int_{a}^{b} \omega(u) \mathrm{d} u\right) \\
& +\int_{a}^{b}\left(\frac{(b+a-2 u)}{(b-a)^{2}}\right)^{2} \mathrm{~d} u \Theta^{T} R \Theta \\
& -2 \Theta^{T} R \int_{a}^{b}\left(\frac{b+a-2 u}{(b-a)^{2}}\right) \omega(u) \mathrm{d} u \\
& +2 \int_{a}^{b}\left(\frac{(b+a-2 u)}{(b-a)^{2}}\right) \mathrm{d} u \Theta^{T} R\left(\int_{a}^{b} \omega(u) \mathrm{d} u\right) .
\end{aligned}
$$

By noting that $\int_{a}^{b}(b+a-2 u) \mathrm{d} u=0$ and by use of an integration by parts, it yields

$$
\begin{gathered}
\int_{a}^{b} \dot{z}^{T}(u) R \dot{z}(u) \mathrm{d} u=\int_{a}^{b} \omega^{T}(u) R \omega(u) \mathrm{d} u \\
-\frac{1}{b-a}\left(\int_{a}^{b} \omega(u) \mathrm{d} u\right)^{T} R\left(\int_{a}^{b} \omega(u) \mathrm{d} u\right) \\
+\frac{1}{3(b-a)} \Theta^{T} R \Theta+\frac{2}{(b-a)} \Theta^{T} R \Omega .
\end{gathered}
$$

Rewriting the two last terms as a sum of squares leads to

$$
\begin{aligned}
\int_{a}^{b} \dot{z}^{T}(u) R \dot{z}(u) \mathrm{d} u=\int_{a}^{b} \omega^{T}(u) R \omega(u) \mathrm{d} u \\
\quad-\frac{1}{b-a}\left(\int_{a}^{b} \omega(u) \mathrm{d} u\right)^{T} R\left(\int_{a}^{b} \omega(u) \mathrm{d} u\right) \\
\quad-\frac{3}{(b-a)} \Omega^{T} R \Omega+\frac{1}{3(b-a)}(\Theta+3 \Omega)^{T} R(\Theta+3 \Omega)
\end{aligned}
$$

Consider now the right-hand side of the inequality (4). Applying the Jensen inequality and noting that $\int_{a}^{b} z(u) \mathrm{d} u=-\frac{(b-a)}{6}(\Theta+3 \Omega)$, Lemma 2 ensures that

$$
\begin{aligned}
& \mathcal{I}_{R}(\omega) \geq \frac{1}{b-a}\left(\int_{a}^{b} \omega(u) \mathrm{d} u\right)^{T} R\left(\int_{a}^{b} \omega(u) \mathrm{d} u\right) \\
& +\frac{3}{(b-a)} \Omega^{T} R \Omega+\left(\frac{\pi^{2}-12}{36(b-a)}\right)(\Theta+3 \Omega)^{T} R(\Theta+3 \Omega) .
\end{aligned}
$$

Since $12 \geq \pi^{2}$, the right-hand side of the previous inequality is definite negative independently of the choice of $\Theta$. Hence, its maximum is reached and is 0 when $\Theta=-3 \Omega$. This concludes the proof.

Remark 1 The previous corollary refines the inequality proposed in [?], in which the last term of the right-handside of (9) is multiplied by $\frac{\pi^{2}}{4}$ which is less than 3 . This proves that the proposed inequality delivers a more accurate lower bound of $\mathcal{I}_{R}(\omega)$ than the one proposed in [?]. 
Remark 2 Since $R \succ 0$, the second term of (9) is definite positive. It thus implies that the Jensen inequality is included in the inequality proposed (5). It is also worth noting that this improvement is allowed by using an extra signal $\int_{a}^{b} \int_{a}^{u} \omega(s) \mathrm{d} s \mathrm{~d} u$ and not only $\int_{a}^{b} \omega(u) \mathrm{d} u$.

As it was mentioned previous section, the differentiation of the Lyapunov-Krasovskii functional proposed in (2) requires to find a lower bound of $\mathcal{I}_{R}(\dot{\omega})$.In such situation, the previous lemma is rewritten as follows.

Corollary 5 For a given matrix $R \succ 0$, the following inequality holds for all continuously differentiable function $\omega$ in $[a, b] \rightarrow \mathbb{R}^{n}$ :

$$
\begin{aligned}
\mathcal{I}_{R}(\dot{\omega}) & \geq \frac{1}{b-a}(\omega(b)-\omega(a))^{T} R(\omega(b)-\omega(a)) \\
& +\frac{3}{(b-a)} \tilde{\Omega}^{T} R \tilde{\Omega},
\end{aligned}
$$

where $\tilde{\Omega}=\omega(b)+\omega(a)-\frac{2}{b-a} \int_{a}^{b} \omega(u) \mathrm{d} u$.

The previous inequality will be employed for the stability analysis of time-delay and sampled-data systems.

\section{Application to the stability analysis of time- delay systems}

\subsection{Systems with constant and known delay}

Consider a linear time-delay system:

$$
\left\{\begin{array}{l}
\dot{x}(t)=A x(t)+A_{d} x(t-h)+A_{D} \int_{t-h}^{t} x(s) d s, \forall t \geq 0, \\
x(t)=\phi(t), \forall t \in[-h, 0],
\end{array}\right.
$$

where $x(t) \in \mathbb{R}^{n}$ is the state vector, $\phi$ is the initial condition and $A, A_{d}, A_{D} \in \mathbb{R}^{n \times n}$ are constant matrices. The delay is assumed to be known and constant. The following stability theorem is provided.

Theorem 6 Assume that, for a given $h>0$, there exist $2 n \times 2 n$-matrix $P \succ 0$, and $n \times n$-matrices $S \succ 0, R \succ 0$ such that the following LMI is satisfied

$$
\Psi(h)=\Psi_{0}(h)-\frac{1}{h} F_{2}^{T} \tilde{R} F_{2} \prec 0,
$$

where

$$
\begin{aligned}
\Psi_{0}(h) & =\operatorname{He}\left(F_{1}^{T}(h) P F_{0}(h)\right)+\bar{S}+h F_{0}^{T}(h) \bar{R} F_{0}(h), \\
F_{0}(h) & =\left[\begin{array}{ccc}
A & A_{d} & h A_{D} \\
I & -I & 0
\end{array}\right], \quad F_{1}(h)=\left[\begin{array}{ccc}
I & 0 & 0 \\
0 & 0 & h I
\end{array}\right], \\
F_{2} & =\left[\begin{array}{ccc}
I & -I & 0 \\
I & I & -2 I
\end{array}\right], \quad \bar{S}=\operatorname{diag}\left(S,-S, 0_{n}\right), \\
\bar{R} & =\operatorname{diag}\left(R, 0_{2 n}\right), \quad \tilde{R}=\operatorname{diag}(R, 3 R),
\end{aligned}
$$

Then the system (10) is asymptotically stable for the constant and known delay $h>0$.

Proof : Consider the functional given by

$$
\begin{aligned}
V\left(h, x_{t}, \dot{x}_{t}\right) & =\bar{x}^{T}(t) P \bar{x}(t)+\int_{t-h}^{t} x^{T}(s) Q x(s) \mathrm{d} s \\
& +\int_{t-h}^{t} \int_{\theta}^{t} \dot{x}^{T}(\theta) R \dot{x}(\theta) \mathrm{d} \theta \mathrm{d} s
\end{aligned}
$$

where $\bar{x}(t)=\left[\begin{array}{c}x(t) \\ \int_{t-h}^{t} x(s) \mathrm{d} s\end{array}\right]$. This functional is positive definite since $P \succ 0, S \succ 0$ and $R \succ 0$. Differentiating (17) along the trajectories of (10) leads to:

$$
\dot{V}\left(h, x_{t}, \dot{x}_{t}\right)=\zeta_{0}^{T}(t) \Psi_{0}(h) \zeta_{0}(t)-\int_{t-h}^{t} \dot{x}^{T}(s) R \dot{x}(s) \mathrm{d} s,
$$

where $\zeta_{0}(t)=\left[x^{T}(t) x^{T}(t-h) \frac{1}{h} \int_{t-h}^{t} x^{T}(s) \mathrm{d} s\right]^{T}$. This equation has been obtained by noting that

$$
\bar{x}(t)=F_{1}(h) \zeta_{0}(t), \dot{\bar{x}}(t)=F_{0}(h) \zeta_{0}(t) .
$$

Then the application of Corollary 5 to the integral defined over the interval $[t-h, t]$ leads to

$$
\int_{t-h}^{t} \dot{x}^{T}(s) R \dot{x}(s) \mathrm{d} s \geq-\frac{1}{h} \zeta_{0}^{T}(t) F_{2}^{T} \tilde{R} F_{2} \zeta_{0}(t)
$$

It yields $\dot{V}\left(h, x_{t}, \dot{x}_{t}\right) \leq \zeta_{0}^{T}(t) \Psi(h) \zeta_{0}(t)$. Then if (11) is satisfied for a given $h>0$, then the system (10) is asymptotically stable for this delay $h$.

Remark 3 It is worth noting that the previous theorem only deals with the case of constant and known delays. It does not mean that the considered system is asymptotically stable for any delay belonging to the interval $[0, h]$.

Remark 4 This case is very special and the case of of different value for the discrete and distributed delays would be more relevant to study. However, the goal of the present article is to show that the two different problems of distributed and discrete delays can be tackled by using a unique class of Lyapunov-Krasovskii functionals.

\subsection{Systems with a time-varying delay}

Consider the following class of systems

$$
\begin{cases}\dot{x}(t)=A x(t)+A_{d} x(t-h(t)), & \forall t \geq 0, \\ x(t)=\phi(t), & \forall t \in[-h, 0],\end{cases}
$$

where the delay function $h$ is unknown or time-varying and satisfies the following constraints

$$
h(t) \in\left[h_{m}, h_{M}\right], \quad \dot{h}(t) \in\left[d_{m}, d_{M}\right], \quad \forall t \geq 0,
$$


where $0 \leq h_{m} \leq h_{M}$ and $d_{m} \leq d_{M} \leq 1$. In such a situation, the following stability theorem is provided.

Theorem 7 Assume that there exist $3 n \times 3 n$-matrix $P \succ$ $0, n \times n$-matrices $S \succ 0, Q \succ 0, R \succ 0$ and $a 2 n \times 2 n$ matrix $X$ such that the following LMIs are satisfied for $h=\left\{h_{m}, h_{M}\right\}$ and for $\dot{h}=\left\{d_{m}, d_{M}\right\}$

$$
\begin{array}{rlr}
\Phi_{1}(h, \dot{h}) & =\Phi_{0}(h, \dot{h})-\frac{1}{h_{M}} \Gamma^{T} \Phi_{2} \Gamma & \prec 0, \\
\Phi_{2} & =\left[\begin{array}{cc}
\tilde{R} & X \\
* & \tilde{R}
\end{array}\right] & \succ 0,
\end{array}
$$

where

$$
\begin{aligned}
& \Phi_{0}(h, \dot{h})=\operatorname{He}\left(G_{1}^{T}(h) P G_{0}(\dot{h})\right)+\hat{S}+\hat{Q}(\dot{h}) \\
& +h_{M} G_{0}^{T}(\dot{h}) \hat{R} G_{0}(\dot{h}), \\
& \Gamma=\left[\begin{array}{llll}
G_{2}^{T} & G_{3}^{T} & G_{4}^{T} & G_{5}^{T}
\end{array}\right]^{T}, \\
& G_{2}=\left[\begin{array}{lllll}
I & -I & 0 & 0 & 0
\end{array}\right], \quad G_{3}=\left[\begin{array}{lllll}
I & I & 0 & -2 I & 0
\end{array}\right] \text {, } \\
& G_{4}=\left[\begin{array}{lllll}
0 & I & -I & 0 & 0
\end{array}\right], \quad G_{5}=\left[\begin{array}{lllll}
0 & I & I & 0 & -2 I
\end{array}\right], \\
& \hat{Q}(\dot{h})=\operatorname{diag}\left(Q,-(1-\dot{h}) Q, 0_{3 n}\right), \\
& \hat{S}=\operatorname{diag}\left(S, 0,-S, 0_{2 n}\right), \\
& \hat{R}=\operatorname{diag}\left(R, 0_{3 n}\right), \quad \tilde{R}=\operatorname{diag}(R, 3 R),
\end{aligned}
$$

and

$$
\begin{aligned}
& G_{0}(\dot{h})=\left[\begin{array}{ccccc}
A & A_{d} & 0 & 0 & 0 \\
I & -(1-\dot{h}) I & 0 & 0 & 0 \\
0 & (1-\dot{h}) I & -I & 0 & 0
\end{array}\right], \\
& G_{1}(h)=\left[\begin{array}{ccccc}
I & 0 & 0 & 0 & 0 \\
0 & 0 & 0 & h I & 0 \\
0 & 0 & 0 & 0 & \left(h_{M}-h\right) I
\end{array}\right] .
\end{aligned}
$$

Then the system (13) is asymptotically stable for all delay function $h$ satisfying (14).

Proof : Consider the functional given by

$$
\begin{aligned}
V\left(h, x_{t}, \dot{x}_{t}\right) & =\tilde{x}^{T}(t) P \tilde{x}(t)+\int_{t-h(t)}^{t} x^{T}(s) Q x(s) \mathrm{d} s \\
& +\int_{t-h_{M}}^{t} x^{T}(s) S x(s) \mathrm{d} s \\
& +\int_{t-h_{M}}^{t} \int_{\theta}^{t} \dot{x}^{T}(s) R \dot{x}(s) \mathrm{d} s \mathrm{~d} \theta
\end{aligned}
$$

where $\tilde{x}(t)=\left[x^{T}(t), \int_{t-h(t)}^{t} x^{T}(s) \mathrm{d} s, \int_{t-h_{M}}^{t-h(t)} x^{T}(s) \mathrm{d} s\right]^{T}$. This functional is positive definite since $P \succ 0, Q \succ 0$, $S \succ 0$ and $R \succ 0$. Differentiating the functional (17) along the trajectories of (13) leads to:

$$
\dot{V}\left(h, x_{t}, \dot{x}_{t}\right)=\zeta_{1}^{T}(t) \Phi_{0}(h) \zeta_{1}(t)-\int_{t-h_{M}}^{t} \dot{x}^{T}(s) R \dot{x}(s) \mathrm{d} s,
$$

where $\zeta_{1}(t)=\left[\begin{array}{c}x(t) \\ x(t-h(t)) \\ x\left(t-h_{M}\right) \\ \frac{1}{h(t)} \int_{t-h(t)}^{t} x(s) \mathrm{d} s \\ \frac{1}{h_{M}-h(t)} \int_{t-h_{M}}^{t-h(t)} x(s) \mathrm{d} s\end{array}\right]^{T}$.

This equation has been obtained by noting that $\tilde{x}(t)=$ $G_{1}(h) \zeta_{1}(t)$ and $\dot{\tilde{x}}(t)=G_{0}(\dot{h}) \zeta_{1}(t)$. The next step consists in splitting the integral into two integrals, taken over the two intervals $\left[t-h_{M}, t-h(t)\right]$ and $[t-h(t), t]$ and in applying Corollary 5 to each of them. It yields

$$
\begin{aligned}
-\int_{t-h_{M}}^{t} \dot{x}^{T}(s) R \dot{x}(s) \mathrm{d} s \leq & -\zeta_{1}^{T}(t)\left(\frac{1}{h(t)} G_{23}^{T} \tilde{R} G_{23}\right. \\
& \left.+\frac{1}{h_{M}-h(t)} G_{45}^{T} \tilde{R} G_{45}\right) \zeta_{1}(t)
\end{aligned}
$$

where $G_{23}=\left[\begin{array}{l}G_{2} \\ G_{3}\end{array}\right]$ and $G_{45}=\left[\begin{array}{l}G_{4} \\ G_{5}\end{array}\right]$. Providing that there exists a matrix $X$ such that $\Phi_{2} \succ 0$, Lemma 3 ensures that

$$
-\int_{t-h_{M}}^{t} \dot{x}^{T}(s) R \dot{x}(s) \mathrm{d} s \leq-\frac{1}{h_{M}} \zeta_{1}^{T}(t) \Gamma^{T} \Phi_{2} \Gamma \zeta_{1}(t),
$$

which leads to $\dot{V}\left(x_{t}, \dot{x}_{t}\right) \leq \zeta_{1}^{T}(t) \Phi(h, \dot{h}) \zeta_{1}(t)$. Finally, $\dot{V}$ is negative definite if there exists a matrix $X$ such that $\Phi_{2} \succ 0$ and if $\Phi(h, \dot{h}) \prec 0$, for all $(h, \dot{h}) \in\left[0, h_{M}\right] \times\left[d_{m}, d_{M}\right]$. Since the matrix $\Phi(h, \dot{h})$ is affine, and consequently convex, with respect to $h(t)$ and $\dot{h}(t)$, it is necessary and sufficient to ensure that $\Phi(h, \dot{h}) \prec 0$ at the vertices of the intervals $\left[0, h_{M}\right] \times\left[d_{m}, d_{M}\right]$, which concludes the proof.

\subsection{Examples}

\subsubsection{Constant distributed delay case}

Consider the linear time-delay systems (10) with the matrices taken from [?]:

$$
A=\left[\begin{array}{cc}
0.2 & 0 \\
0.2 & 0.1
\end{array}\right], A_{d}=\left[\begin{array}{ll}
0 & 0 \\
0 & 0
\end{array}\right], A_{D}=\left[\begin{array}{cc}
-1 & 0 \\
-1 & -1
\end{array}\right]
$$

An eigenvalue analysis provides that the system remains stable for all constant delays in the interval [0.200, 2.04]. In [? ] and [?], stability is guaranteed for delays over the interval $[0.2090,1.1942]$ and $[0.2001,1.6339]$, respectively. Using our new inequality, Theorem 6 ensures stability for all constant delays which belong to the interval $[0.200,1.877]$ which encompasses these previous results and shows the potential of Corollary 5 . 


\begin{tabular}{|l|c|c|c|c|c|c|c|}
\hline$d_{M}\left(=-d_{m}\right)$ & 0 & 0.1 & 0.2 & 0.5 & 0.8 & 1 & $N_{v}$ \\
\hline \hline$[?]$ & 4.472 & 3.604 & 3.033 & 2.008 & 1.364 & 0.999 & $5.5 n^{2}+1.5 n$ \\
\hline$[?]$ & 1.632 & 1.632 & 1.632 & 1.632 & 1.632 & 1.632 & $7 n^{2}+n$ \\
\hline$[? \boldsymbol{?}]$ & 4.472 & 3.605 & 3.039 & 2.043 & 1.492 & 1.345 & $3 n^{2}+3 n$ \\
\hline$[?]$ & 4.472 & 3.611 & 3.047 & 2.072 & 1.590 & 1.529 & $7 n^{2}+n$ \\
\hline$[?][\mathrm{N}=1)$ & 6.059 & $-{ }^{1}-$ & $-^{1}$ & $-{ }^{1}$ & $-{ }^{1}$ & $-{ }^{1}$ & $5.5 n^{2}+2.5 n$ \\
\hline$[?]$ & 6.117 & 4.714 & 3.807 & 2.280 & 1.608 & 1.360 & $1.5 n^{2}+9 n+9$ \\
\hline$[?]$ & 6.117 & 4.794 & 3.995 & 2.682 & 1.957 & 1.602 & $22 n^{2}+8 n$ \\
\hline \hline Th.6 & 6.059 & $-{ }^{1}$ & $-{ }^{1}$ & $-{ }^{1}$ & $-{ }^{1}$ & $-{ }^{1}$ & $3 n^{2}+2 n$ \\
\hline Th.7 & 6.059 & 4.703 & 3.834 & 2.420 & 2.137 & 2.128 & $10 n^{2}+3 n$ \\
\hline
\end{tabular}

Table 1

The maximal allowable delays $h_{M}$ for system described in Example (20).

\subsubsection{Unknown time-varying delay case}

We consider the linear time-delay systems (13) with

$$
A=\left[\begin{array}{cc}
-2 & 0 \\
0 & -0.9
\end{array}\right], A_{d}=\left[\begin{array}{cc}
-1 & 0 \\
-1 & -1
\end{array}\right], A_{D}=\left[\begin{array}{ll}
0 & 0 \\
0 & 0
\end{array}\right]
$$

This system is a well-known delay dependent stable system where the maximum allowable delay $h_{\max }=6.1721$ can be easily computed by delay sweeping techniques. To demonstrate the effectiveness of our approach, results are compared to the literature and are reported in Table 1. Table 1 shows that our result is competitive with the most accurate stability conditions from the literature. For the case of constant and known delay, Theorem 6 delivers the same result as the one provided by the discretization method $^{1}$ of [?] with $N=1$ and with a lower number of variables. For the time-varying case, only the conditions provided in [?] and in [?] are less conservative than the ones of Theorem 7 for slow varying delays. However for fast varying delays, Theorem 7 becomes less conservative than the conditions from these articles.

\section{Application to sampled-data systems}

Let $\left\{t_{k}\right\}_{k \in \mathbb{N}}$ be an increasing sequence of positive scalars such that $\bigcup_{k \in \mathbb{N}}\left[t_{k}, t_{k+1}\right)=[0,+\infty)$, for which there exist two positive scalars $T_{\min } \leq T_{\max }$ such that

$$
\forall k \in \mathbb{N}, \quad T_{k}=t_{k+1}-t_{k} \in\left[T_{\min }, T_{\max }\right] .
$$

The sequence $\left\{t_{k}\right\}_{k \in \mathbb{N}}$ represents the sampling instants. Consider the sampled-data system given by

$$
\forall t \in\left[t_{k}, t_{k+1}\right), \quad \dot{x}(t)=A_{c} x(t)+A_{s} x\left(t_{k}\right),
$$

where $x \in \mathbb{R}^{n}$ represents the state. The matrices $A_{c}$ and $A_{s}$ are constant, known and of appropriate dimensions.

1 The stability conditions providedby this method only concerns the case of constant and known delays
Adopting the method based on looped-functionals proposed in [? ? ?], the following result is proposed

Theorem 8 Let $0<T_{\min } \leq T_{\max }$ be two positive scalars. Assume that there exist $n \times n$-matrices $P \succ 0$, $R \succ 0, S=S^{T}, Q=Q^{T}$ and $X=X^{T}$ and $3 n \times n-$ matrices $Y_{1} Y_{2}$ and $Y_{3}$ that satisfy

$$
\begin{gathered}
\Theta_{1}(T)=\Pi_{1}+T\left(\Pi_{2}+\Pi_{3}\right) \\
\Theta_{2}(T)=\left[\begin{array}{ccc}
\Pi_{1}-T\left(\Pi_{3}+\Pi_{4}\right) & T Y_{1} & 3 T Y_{2} \\
* & -T R & 0 \\
* & * & -3 T R
\end{array}\right] \prec 0,
\end{gathered}
$$

for $T \in\left\{T_{\min }, T_{\max }\right\}$ and where

$$
\begin{aligned}
& \Pi_{1}=\Pi_{1}^{0}-\operatorname{He}\left\{\left(Y_{1}+Y_{3}\right) W_{1}+3 Y_{2} W_{2}\right\} \\
& \Pi_{1}^{0}=\operatorname{He}\left\{M_{1}^{T} P M_{0}-W_{1}^{T} Q M_{2}\right\}-W_{1}^{T} S W_{1}, \\
& \Pi_{2}=M_{0}^{T} R M_{0}+\operatorname{He}\left\{M_{0}^{T} S W_{1}+M_{0}^{T} Q M_{2}\right\}, \\
& \Pi_{3}=M_{2}^{T} X M_{2}, \quad \Pi_{4}=Y_{3} M_{4},
\end{aligned}
$$

with $M_{0}=\left[\begin{array}{lll}A_{c} & A_{s} & 0\end{array}\right], M_{1}=\left[\begin{array}{lll}I & 0 & 0\end{array}\right], M_{2}=\left[\begin{array}{lll}0 & I & 0\end{array}\right]$, $M_{3}=\left[\begin{array}{lll}0 & 0 & I\end{array}\right], M_{4}=\left[\begin{array}{lll}0 & A_{s} & A_{c}\end{array}\right], W_{1}=\left[\begin{array}{lll}I & -I & 0\end{array}\right]$ and $W_{2}=\left[\begin{array}{ll}I & I\end{array}-2\right]$. Then the system $(22)$ is asymptotically stable for all sequence $\left\{t_{k}\right\}_{k \geq 0}$ satisfying (21).

Proof : Consider a given integer $k \geq 0$ and the associated $T_{k} \in\left[T_{\min } T_{\max }\right]$. The stability analysis can be performed using the quadratic function $V(x)=x^{T} P x$ where $P \succ 0$ and a functional $\mathcal{V}_{0}$ of the form

$$
\begin{aligned}
\mathcal{V}_{0}\left(t-t_{k}, x\right) & =\left(t_{k+1}-t\right) \tilde{x}^{T}(t)\left(S \tilde{x}(t)+2 Q x\left(t_{k}\right)\right) \\
& +\left(t_{k+1}-t\right) \int_{t_{k}}^{t} \dot{x}^{T}(s) R \dot{x}(s) \mathrm{d} s \\
& +\left(t_{k+1}-t\right)\left(t-t_{k}\right) x^{T}\left(t_{k}\right) X x\left(t_{k}\right),
\end{aligned}
$$

where $\tilde{x}(t)=x(t)-x\left(t_{k}\right)$. This functional is called a looped functionals because it satisfies the boundary conditions $\mathcal{V}_{0}(0, x)=\mathcal{V}_{0}\left(T_{k}, x\right)=0$. Define the functional $\mathcal{W}=V+\mathcal{V}_{0}$ (see [? ? ? ] for more details). In 
the following, the notation $\tau=t-t_{k}$ is adopted. Introduce the vectors $\nu_{k}(\tau)=\frac{1}{\tau} \int_{t_{k}}^{t} x(s) \mathrm{d} s$ and $\xi_{k}(\tau)=$ $\left[x^{T}(t) x^{T}\left(t_{k}\right) \nu_{k}^{T}(\tau)\right]$. Since the vector $x\left(t_{k}\right)$ is constant over $\left[\begin{array}{ll}t_{k} & t\end{array}\right] \subset\left[\begin{array}{ll}t_{k} & t_{k+1}\end{array}\right]$, the following equation is derived, for all matrix $Y_{3} \in \mathbb{R}^{3 n \times n}$

$$
\begin{aligned}
2 \xi_{k}^{T}(\tau) Y_{3} \int_{t_{k}}^{t} \dot{x}(s) \mathrm{d} s & =2 \xi_{k}^{T}(\tau) Y_{3}\left(x(t)-x\left(t_{k}\right)\right) \\
& =2 \tau \xi_{k}^{T}(\tau) Y_{3} M_{4} \xi_{k}(\tau)
\end{aligned}
$$

This expression shows that there exists a relation between the vectors $x(t), x\left(t_{k}\right)$ and $\nu_{k}(\tau)$. Hence, following the proof of Theorem 2 in [?], the computation of the derivative of $\mathcal{W}$ together with the linking relation (25)

$$
\begin{aligned}
\dot{\mathcal{W}}(\tau, x)= & \xi_{k}^{T}(\tau)\left[\Pi_{1}^{0}+\left(T_{k}-\tau\right) \Pi_{2}+\left(T_{k}-2 \tau\right) \Pi_{3}\right. \\
& \left.+\tau \Pi_{4}\right] \xi_{k}(\tau)-\int_{t_{k}}^{t} \dot{x}^{T}(s) R \dot{x}(s) \mathrm{d} s .
\end{aligned}
$$

Applying Corollary 5 yields

$$
\begin{aligned}
& -\int_{t_{k}}^{t} \dot{x}^{T}(s) R \dot{x}(s) \mathrm{d} s \leq \\
& \quad-\frac{1}{\tau} \xi_{k}^{T}(\tau)\left[W_{1}^{T} R W_{1}+3 W_{2}^{T} R W_{2}\right] \xi_{k}(\tau)
\end{aligned}
$$

Noting that, for all matrices $Y_{i}, i \in\{1,2\}$ in $\mathbb{R}^{n \times 3 n}$, it holds $\frac{1}{\tau}\left(R W_{i}-\tau Y_{i}\right)^{T} R^{-1}\left(R W_{i}-\tau Y_{i}\right) \succ 0$ for all $i \in\{1,2\}$, the inequality

$$
-\frac{1}{\tau} W_{i}^{T} R W_{i} \leq-Y_{i}^{T} W_{i}-W_{i}^{T} Y_{i}+\tau Y_{i}^{T} R^{-1} Y_{i}
$$

holds for $i=1,2$. This ensures

$$
\dot{\mathcal{W}}(\tau, x)=\xi_{k}^{T}(\tau) \Pi\left(\tau, T_{k}\right) \xi_{k}(\tau)
$$

where $\Pi\left(\tau, T_{k}\right)=\Pi_{1}+\left(T_{k}-\tau\right) \Pi_{2}+\left(T_{k}-2 \tau\right) \Pi_{3}+\tau \bar{\Pi}_{4}$ and $\bar{\Pi}_{4}=\Pi_{4}+Y_{1} R^{-1} Y_{1}^{T}+3 Y_{2} R^{-1} Y_{2}^{T}$. Since $\Pi\left(\tau, T_{k}\right)$ is affine, and consequently convex, with respect to $\tau \in\left[0, T_{k}\right]$, it is sufficient to ensures that $\Pi\left(0, T_{k}\right) \prec 0$ and $\Pi\left(T_{k}, T_{k}\right) \prec 0$, or equivalently $\Theta_{1}\left(T_{k}\right) \prec 0$ and $\Theta_{2}\left(T_{k}\right) \prec 0$. The same argument on the parameter $T_{k} \in\left[T_{\min } T_{\max }\right]$ ensures that $\dot{\mathcal{W}} \leq 0$. According to [? ], the system (22) is asymptotically stable.

\subsection{Examples}

Consider again the system (22) provided in the example (20) with $A_{c}=A$ and $A_{s}=A_{d}$. Additionally, we will also consider the following example taken from [? ? ].

$$
\dot{x}(t)=\left[\begin{array}{cc}
0 & 1 \\
0 & -0.1
\end{array}\right] x(t)+\left[\begin{array}{c}
0 \\
-0.1
\end{array}\right]\left[\begin{array}{c}
3.75 \\
11.5
\end{array}\right]^{T} x\left(t_{k}\right)
$$

\begin{tabular}{|l|l|l|l|}
\hline Theorems & Ex. $(20)$ & Ex. $(26)$ & Nb of variables \\
\hline \hline Th. bounds & $(0,3.269]$ & $(0,1.729]$ & - \\
\hline$[\boldsymbol{?}]$ & $(0,2.53]$ & $(0,1.695]$ & $8 n^{2}+n$ \\
\hline$[?]$ & $(0,2.53]$ & $(0,1.695]$ & $11.5 n^{2}+2.5 n$ \\
\hline$[?]$ & $(0,2.62]$ & $(0,1.721]$ & $5 n^{2}+2 n$ \\
\hline \hline Th. 8 & $(0,2.87]$ & $(0,1.729]$ & $12 n^{2}+3 n$ \\
\hline
\end{tabular}

\section{Table 2}

Interval of allowable asynchronous samplings. The theoretical bounds have been computed by an eigenvalue analysis for the case of synchronous samplings.

For this example, when the sampling period is chosen constant (i.e. $T_{k}=T_{\min }=T_{\max }$, for all $k \geq 0$ ), an eigenvalue analysis of the transition matrix ensures that the system is stable for all constant sampling period in (0, 1.729]. Applying Theorem 8, we prove that system (26) is asymptotically stable for all asynchronous sampling over the interval $[0,1.724]$, encompassing many results of the literature as it can be seen in the Table 2 but at a increasing numerical burden.

\section{Conclusions}

In this paper, we have provided a new inequality which encompasses the Jensen inequality. In combination with a simple choice of Lyapunov-Krasovskii functionals, this inequality leads to new stability criteria for linear timedelay and sampled-data systems. These new results have been expressed in terms of LMIs and has shown on numerical examples large improvements of existing results using only a limited number of matrix variables. 